\title{
UN MODELO MICROECONOMETRICO PARA EL ANALISIS DE INTEGRACIONES EMPRESARIALES: EL CASO DEL MERCADO DE BEBIDAS ISOTONICAS*
}

\author{
A MICROECONOMETRIC MODEL FOR THE ANALYSIS OF MERGERS: \\ THE CASE OF SPORTS DRINKS
}

\section{DENNIS SANCHEZ NAVARRO**}

Banco Interamericano de Desarrollo, Estados Unidos

\section{JUAN PABLO HERRERA SAAVEDRA ${ }^{\star \star \star}$}

Superintendencia de Industria y Comercio, Colombia

\section{AURA GARCIA PABON****}

Superintendencia de Industria y Comercio, Colombia

\section{JACOBO CAMPO ROBLEDO*****}

Superintendencia de Industria y Comercio, Colombia

* Los autores agradecen a los editores y a los evaluadores anónimos, cuyas observaciones permitieron mejorar sustancialmente el documento, al Grupo de Integraciones Empresariales de la Superintendencia de Industria y Comercio, y a los asistentes a los diferentes congresos en los que se presentaron los resultados de este documento. Las opiniones e interpretaciones en este documento son estrictamente las de los autores y no deben atribuirse al Banco Interamericano de Desarrollo, su Junta Directiva o los países que representan, ni a la Superintendencia de Industria y Comercio, ni a ninguna de las Instituciones.

** Durante la elaboración de este documento se desempeñaba como Economista del Grupo de Estudios Económicos de la Superintendencia de Industria y Comercio. Ahora se desempeña como consultor económico para asuntos fiscales y laborales en el Banco Interamericano de Desarrollo. E-mail: dennis.seni@gmail.com o denniss@iadb.org

*** Superintendente Delegado para la Protección de la Competencia, Profesor de Economía Facultad de Ciencias Económicas Universidad Nacional de Colombia y Facultad de Economía Universidad Externado de Colombia.. E-mail: jpherrera@sic.gov.co

**** Msc Economía, competencia y regulación de la Universidad de Tilburg - Países Bajos y economista de la Universidad del Rosario. Asesora del Superintendente de Industria y Comercio y profesora de las facultades de economía de la Universidad Externado de Colombia y de la Pontificia Universidad Javeriana. E-mail: amgarcia@sic.gov.co

***** Coordinador del Grupo de Estudios Económicos, Superintendencia de Industria y Comercio. Profesor de las Facultades de Economía en las Universidades Católica de Colombia y Externado de Colombia. E-mail: jcampo@sic.gov.co 


\begin{abstract}
This document presents an analysis of the effect of mergers on competition and prices, in the framework of a merger between two representative isotonic drinks producers in 2014-2015. In particular, between Brand 3 and Brand 4. Given the descriptive analysis and the econometric excercise presented, it is possible to conclude that there is no evidence of a possible exploitative effect in the market as no price-increasing pressures were found as a result of the merger. This documents constitutes a relevant microeconometric tool to be used for the analysis of mergers in Colombia.
\end{abstract}

Keywords: Mergers, competition, exploitative effect, logit model.

JEL Classification: $C 23, C 26, D 40$.

\title{
Resumen
}

En este documento se lleva a cabo un análisis del efecto sobre la competencia y los precios que pueden derivarse de una integración empresarial, en el marco de la integración empresarial propuesta entre dos grandes productoras de bebidas isotónicas en el año 2014-2015. En particular, se evalúa el posible impacto que pudiera derivarse de la integración en el mercado en mención, particularmente entre los productos Marca 3 y Marca 4. Tras el análisis descriptivo y el ejercicio econométrico, es posible concluir que no se encuentran indicios sobre un posible efecto explotativo explicado la inexistencia de presiones al alza en precios como resultado de la posible integración. El documento se constituye en una herramienta microeconométrica en el análisis de integraciones empresariales en Colombia.

Palabras clave: Integración empresarial, competencia, efecto explotativo, modelo logit.

Clasificación JEL: C23, C26, D40.

\section{INTRODUCCION}

La integración empresarial ha sido un mecanismo ampliamente utilizado a nivel mundial por las empresas cuando quieren adquirir el control de una o varias empresas con el objetivo de desarrollar actividades conjuntamente. En definitiva, una integración es una combinación de una o más actividades que desempeñan las empresas intervinientes en el proceso, entre las cuales cesa cualquier mecanismo de competencia. 
El artículo 9 sobre el control de Integraciones Empresariales de la Ley 1340 del 24 de Julio de 2009 que modificó el artículo $4^{\circ}$ de la Ley 155 de 1959 sostiene que

"Las empresas que se dediquen a la misma actividad económica o participen en la misma cadena de valor, y que cumplan con las siguientes condiciones, estarán obligadas a informar a la Superintendencia de Industria y Comercio sobre las operaciones que proyecten llevar a cabo para efectos de fusionarse, consolidarse, adquirir el control o integrarse cualquiera sea la forma jurídica de la operación proyectada:

1. Cuando, en conjunto o individualmente consideradas, hayan tenido durante el año fiscal anterior a la operación proyectada ingresos operacionales superiores al monto que, en salarios mínimos legales mensuales vigentes, haya establecido la Superintendencia de Industria y Comercio, o

2. Cuando al finalizar el año fiscal anterior a la operación proyectada tuviesen, en conjunto o individualmente consideradas, activos totales superiores al monto que, en salarios mínimos legales mensuales vigentes, haya establecido la Superintendencia de Industria y Comercio.

En los eventos en que los interesados cumplan con alguna de las dos condiciones anteriores, pero en conjunto cuenten con menos del $20 \%$ mercado relevante, se entenderá autorizada la operación. Para este último caso se deberá únicamente notificar a la Superintendencia de Industria y Comercio de esta operación”.

En resumen, cuando dos o más empresas se encuentran considerando la posibilidad de integrarse, ya sea vertical u horizontalmente, deben consultar a la Superintendencia de Industria y Comercio para que esta entidad autorice dicha integración.

Dada la importancia de esta práctica, tanto para los productores y los diseñadores de política económica, como para los consumidores, las simulaciones sobre integraciones empresariales han servido como una herramienta guía que permite evaluar los efectos unilaterales de una integración sobre la competencia. Algunos autores han mostrado la importancia de esta herramienta, y el rápido crecimiento que su implementación ha tenido a nivel mundial. Entre otros, Epstein y Rubinfeld (2001) presentan una descripción simple de los principios que debe tener una simulación de integración.

En este orden de ideas, en el presente documento se lleva a cabo un análisis del efecto sobre la competencia y los precios que pueden derivarse de una integración empresarial, en el marco de la integración propuesta entre dos importantes empresas en el año 2014 en torno al mercado de bebidas isotónicas ${ }^{1}$. En particular, el presente documento evalúa el posible impacto que pudiera derivarse de la integración en

1 Con el fin de prevenir revelar información sensible, se anonimizó la información de las empresas activas en el mercado analizado, por lo cual se asignó un número de identificación aleatoriamente tanto a las empresas como a sus marcas. 
el mercado en mención, particularmente entre los productos Marca 3 y Marca 4, producidos por las Firmas 3 y 4, respectivamente.

La valoración del posible efecto sobre la competencia y las principales variables del mercado de bebidas isotónicas se llevó a cabo a partir de dos componentes. El primero, corresponde a un análisis descriptivo sobre el comportamiento de las variables de precios y cantidades vendidas como variables indicativas de la dinámica y contestabilidad del mercado. En segundo lugar, se realiza un ejercicio econométrico a partir del cual se estima la elasticidad de la demanda (propia y cruzada), así como el efecto esperado en los precios por mililitro ante la posible integración.

Con el fin de tener una presentación comparable entre las diferentes marcas que fuera representativa de la dinámica de cada firma, se tomó como referencia del ejercicio la presentación PET de 500ml.

Este documento se encuentra organizado de la siguiente manera: en la segunda sección se presenta una revisión de literatura sobre metodologías que miden los efectos de las integraciones empresariales. En la tercera sección, se presenta un breve análisis de los hechos estilizados del mercado de bebidas isotónicas, la metodología econométrica empleada en el análisis, así como los datos utilizados y su fuente. En la cuarta sección se presenta Las estimaciones y los resultados. Finalmente, en la quinta sección se presentan las conclusiones del ejercicio.

\section{REVISION DE LITERATURA}

La literatura económica sobre los efectos en materia de competencia de las operaciones de integración empresarial incluye análisis tanto teórico como empírico y enfatiza en la necesidad de cuantificar sus efectos sobre niveles de precio, bienestar y concentración de los mercados. Budzinski y Ruhmer (2009) proponen un esquema de clasificación de los mecanismos de análisis propuestos en la literatura que los agrupa en dos etapas. La primera etapa de clasificación corresponde a la forma en la que los agentes interactúan en el mercado y la variable a determinar por las firmas y la segunda se refiere a la forma de la función de demanda del mercado a considerar. En este sentido, dos grupos de modelos son de especial relevancia para el presente documento: los modelos de competencia en cantidades (Cournot) que son un referente teórico sobre los efectos de las integraciones empresariales en los niveles de precios y bienestar, y los modelos de competencia en cantidades que permiten usar herramientas econométricas robustas para realizar estimaciones de demanda.

Farrell y Shapiro (1990) analizan el efecto de integraciones empresariales horizontales sobre los niveles de precio y de bienestar total de la economía. Para esto, desarrollan un modelo de oligopolio de Cournot y encuentran condiciones sobre las cuales las firmas integradas tienen incentivos para aumentar sus precios en función de las sinergias creadas en la estructura de costos. El primer resultado del documento mencionado es que el margen entre el nivel de precios y los costos de la 
firma integrada debe ser alto para que el nivel de precios en el mercado baje. Esto sucede porque la firma integrada debe tener costos marginales lo suficientemente bajos para que superen el incentivo extra de reducir su producción después de realizada la operación de integración. En este sentido, los autores encuentran que no es suficiente para la firma relocalizar su producción a la facilidad más eficiente, sino que, postintegración, los costos marginales de la firma deben reducirse. En cuanto a los cambios en el bienestar total de la economía, Farrel y Shapiro encuentran que estos dependen netamente en el cambio en las cantidades producidas por las firmas involucradas en la operación, y, que el efecto total será positivo, si las firmas rivales son muy sensibles a los cambios en las cantidades de las firmas integradas o si estas últimas no tenían una participación de mercado muy grande en un principio.

Otro documento que analiza teóricamente los efectos de las integraciones empresariales en el bienestar de los consumidores es De Bettignies y Ross (2014). En este documento, los autores presentan un modelo de duopolio de Cournot en donde los gerentes de las empresas realizan inversiones para reducir costos. El principal resultado del documento consiste en que, cuando no existen costos de agencia, la integración empresarial en este escenario llevaría a mayores niveles de inversión y a una reducción en los costos de producción y, en este sentido, a mayores niveles de bienestar social.

Por otro lado, Ivaldi y Verboven (2005) desarrollan un modelo oligopolístico con bienes diferenciados en el que se basan para realizar un estudio comparativo de medidas de poder de mercado: Un test de poder de mercado hipotético, un test de poder de mercado actual y un test de poder de mercado comparativo. Según los autores, este último test muestra que, en una evaluación de integraciones empresariales, un escenario alternativo relevante a considerar es aquel que sucedería si la operación no es aprobada.

Finalmente, documentos como Froeb y Werden (1994) y Froeb et al. (2003) utilizan modelos de competencia en precios con formas específicas de demanda para realizar estimaciones de elasticidades de la demanda con respecto a los precios y poder simular los posibles efectos de las integraciones empresariales sobre los precios y los niveles de competencia en los mercados. En el primer ejercicio, los autores asumen un escenario de equilibrio en precios en un mercado de productos diferenciados y costos marginales constantes y utilizan un modelo logit para predecir efectos sobre precios y bienestar de integraciones empresariales en el sector de telecomunicaciones de larga distancia en Estados Unidos. El resultado principal de la simulación es que solo aquellas operaciones que involucran a la firma AT\&T disminuirían significativamente el bienestar en la economía. Para el segundo documento mencionado, los autores simulan la integración de agentes en el mercado de parqueaderos para autos con el fin de analizar los efectos de dicha integración en un mercado en el que las firmas enfrentan restricciones de capacidad. Para tal fin, utilizan un modelo logit con bienes diferenciados por la distancia de viaje entre el parqueadero y el destino final de los consumidores. El resultado del ejercicio sugiere que las restricciones de capacidad 
que enfrentan los espacios de estacionamiento son más relevantes sobre las empresas que se pretenden integrar que en sus rivales para la determinación de los efectos de competencia de la operación.

Nevo (2000) discute una alternativa para el análisis de las integraciones empresariales cuando la integración ocurre entre empresas de industrias con productos diferenciados, que consiste en empelar la estimación de la demanda y un modelo de conducta después de la integración que recogen los efectos sobre la competencia del mercado.

Finalmente, documentos como Björnerstedt, y Verboven (2016) analizan una gran integración en el mercado de los analgésicos empleando una simulación de integraciones propuesta en Björnerstedt, y Verboven (2013). Sus resultados muestran que la simulación de la integración analizada predice un incremento de los precios de la empresa más pequeña, evidenciando un efecto después de la integración materializado en el aumento de los costos.

Resultados como los mencionados son de gran importancia en el ejercicio de la política de competencia y del análisis de los efectos de las integraciones empresariales, por lo que desarrollar una herramienta que cuantifique los efectos que en el mercado tendría una integración empresarial, se constituye como una prioridad para la autoridad de competencia ${ }^{2}$.

\section{METODO ECONOMETRICO Y DATOS}

Con el fin de validarlos efectos que se podrían derivar de la integración entre las Firmas 3 y 4, en esta sección se procede a realizar una estimación econométrica de las elasticidades (precio propio y cruzada) de la demanda de estos productos. Inicialmente se presenta un análisis descriptivo, seguido de la metodología empleada y posteriormente una descripción de los datos empleados.

\subsection{Análisis inicial}

Si se estima el coeficiente de correlación entre los precios y los volúmenes de ventas (en mililitros), se encuentra que el comportamiento de las ventas del producto Marca 3 es el que muestra el menor nivel de correlación con respecto a lo observado en los precios, con un nivel de correlación inferior al 10\%, mientras que la demanda de otros productos como Marca 2 y Marca 4 resulta mucho más sensible a lo observado en la variable de precios al presentar niveles de correlación mayores al 98\%. Estos resultados se presentan a continuación en el Tabla 1.

2 Es importante reconocer que la metodología de estimación a utilizar en el presente ejercicio es apenas una de múltiples existentes, entre las cuales se resalta el trabajo de Deaton y Muelbauer (1980), que resulta útil en aquellos escenarios en los que se cuenta con un conjunto más amplio de información. 


\section{TABLA 1}

COEFICIENTES DE CORRELACION

\begin{tabular}{|c|r|}
\hline Marca 1 & $-31.7 \%$ \\
\hline Marca 2 & $-98.3 \%$ \\
\hline Marca 3 & $6.9 \%$ \\
\hline Marca 4 & $-98.4 \%$ \\
\hline
\end{tabular}

Fuente: Cálculos GEE-SIC.

En resumen, la respuesta de la demanda de los productos Marca 1, Marca 2 y Marca 4, presenta una mayor correlación con respecto a lo observado en la serie de precios que Marca 3, lo que de forma preliminar podría relacionarse con una elasticidad reducida de éste último ante las variaciones en precios y un comportamiento aparentemente independiente a lo reportado en otros productos del mercado de bebidas isotónicas.

\subsection{Metodología de simulación}

Siguiendo a Björnerstedt y Verboven (2013), si consideramos un mercado con $J$ productos, la función de demanda del producto $j$ es $q_{j}(\mathbf{p})$, siendo $\mathbf{p}$ un vector columna de precios de dimensión $J x 1, c j$ representa su costo marginal constante

$$
\Pi_{f}(\mathbf{p})=\sum_{j \in F_{f}}\left(p_{j}-c_{j}\right) q_{j}(\mathbf{p})+\sum_{j \notin F_{f}}\left(p_{j}-c_{j}\right) q_{j}(\mathbf{p})
$$

Donde las firmas tienen una perfecta coordinación y actúan como un cartel que maximiza beneficios. En este orden de ideas, el equilibrio de Bertrand-Nash está definido por las siguientes condiciones de primer orden

$$
\frac{\partial \Pi_{f}(\mathbf{p})}{\partial p_{j}}=q_{j}(\mathbf{p})+\sum_{k \in F_{f}}\left(p_{k}-c_{k}\right) \frac{\partial q_{k}(\mathbf{p})}{\partial p_{j}}+\sum_{k \notin F_{f}}\left(p_{k}-c_{k}\right) \frac{\partial q_{k}(\mathbf{p})}{\partial p_{j}}=0
$$

Ahora, si se denota a $\theta$, como la matriz de producto de dimensiones $J x J, \theta=\mathbf{1}$ si los productos $j$ y $k$ son producidos por la misma firma. Si denotamos $\mathbf{q}(\mathbf{p})$ como el vector de demanda de dimensión $J x l$, el cambio en el precio $(\Delta \mathbf{p})$ será equivalente a matriz Jacobiana $J x J$ de la primera derivada $\frac{\partial \boldsymbol{q}(\boldsymbol{p})}{\partial \boldsymbol{p}^{\prime}}$, y por último el costo marginal c es un vector $J x l$. En notación vectorial se tiene 


$$
\boldsymbol{q}(\boldsymbol{p})+\{\theta \odot \Delta(\boldsymbol{p})\}(\boldsymbol{p}-\boldsymbol{c})=0
$$

Esta expresión puede invertirse para escribirla como

$$
\boldsymbol{p}=\boldsymbol{c}-\{\theta \odot \Delta(p)\}^{-1} \boldsymbol{q}(\boldsymbol{p})
$$

En el caso de firmas multi producto y/o en colusión, la elasticidad precio cruzada tiene mucha importancia en el mark-up ya que lo incrementa, suponiendo que los productos son sustitutos.

La ecuación (4) puede emplearse para dos finalidades, la primera consiste en reescribirla como el costo marginal pre integración basada en los precios antes de la integración, para estimar las elasticidades precio de la demanda

$$
c^{\text {Pre }}=p^{\text {Pre }}+\left\{\theta^{\text {Pre }} \odot \Delta\left(p^{\text {Pre }}\right)\right\}^{-1} \mathbf{q}\left(p^{\text {Pre }}\right)
$$

Y segundo, puede ser empleada para predecir el equilibrio de Nash después de la integración. Esta operación de integración entre dos empresas implica dos posibles cambios en el mercado, el primero es un cambio en la matriz de producto, de $\boldsymbol{\theta}^{\text {Pre }}$ a $\boldsymbol{\theta}^{\text {Post }}$, y segundo en el caso de existir eficiencias, un cambio en el costo marginal de $\mathbf{c}^{\text {Pre }}$ a $\mathbf{c}^{\text {Post }}$.

Para simular el precio de equilibrio después de la integración se emplea una iteración del punto fijo de la ecuación (4).

\subsection{Metodología}

En esta sección se presentan tres modelos econométricos con el fin de analizar los posibles efectos que sobre el mercado de bebidas isotónicas pudieran derivarse de la integración asociada a los productos Marca 3 y Marca 4. La estimación de estos modelos sigue de cerca la metodología propuesta por Berry (1994), y mejorada por Björnerstedt y Verboven (2013).

En particular, como se indica en Sánchez (2013), la metodología de Berry resulta de gran utilidad en tanto permite obtener la estimación de las elasticidades, partiendo de una descripción del modelo de maximización de utilidad del agente representativo y transformando dicho problema en una descomposición de parámetro de gustos, que permite expresar el nivel medio de utilidad de forma lineal y adicionalmente trabajar con las participaciones de mercado para adelantar la estimación.

El modelo originalmente propuesto por Berry (1994) es el siguiente:

$$
\text { (1) } \ln \left(\frac{s_{j}}{s_{0}}\right)=x_{j} \beta-\alpha p_{j}+\xi_{j}
$$


Donde, sj corresponde a la participación de mercado del bien $j$ en relación al mercado potencial del producto. $s_{0}$ corresponde a la participación de mercado del bien "outside good" en la literatura que permite incorporar la opción de no compra por parte del consumidor. Esta variable se expresa en términos de participación con respecto al mercado potencial del producto. $x_{j}$ es la matriz de elementos exógenos o características propias del producto que inciden como factores diferenciadores para el consumidor. $p_{j}$ es el vector de precios y $\xi_{j}$ es el término de error del modelo a estimar.

De esa forma, los parámetros a estimar serían $\beta$ y $\alpha$. Siendo $\alpha$ el coeficiente de mayor interés para la estimación de las elasticidades precio propias y cruzadas. Berry (1994) estableció que a partir de los resultados del modelo descrito en la ecuación (1), se pueden construir las elasticidades precio-propia y precio-cruzada de la demanda, como se expone a continuación.

La elasticidad-precio del bien $j$ sería:

$$
\text { (2) } \eta_{j}=\frac{\partial s_{j}}{\partial p_{j}} * \frac{P_{j}}{s_{j}}=-\alpha s_{j}\left(1-s_{j}\right) * \frac{P_{j}}{s_{j}}
$$

Mientras que la elasticidad precio-cruzada de la demanda corresponde a:

$$
\eta_{j k}=\frac{\partial s_{j}}{\partial p_{k}} * \frac{P_{k}}{s_{j}}=\alpha s_{j} s_{k} * \frac{P_{k}}{s_{j}}
$$

Teniendo en cuenta lo anterior, y con el fin de estimar la elasticidad propia y elasticidad cruzada entre 4 marcas de bebidas isotónicas se propuso el siguiente modelo:

$$
\text { (4) } \ln \left(\frac{s_{j}}{s_{0}}\right)=\beta_{0}-\alpha p_{j}+\xi_{j}
$$

Sin embargo, debido a los problemas de endogeneidad que podrían presentarse si se realiza la estimación incorporando directamente la variable de precios, se optó por utilizar tres posibles instrumentos disponibles en la base de datos ${ }^{3}$. Estos instrumentos, así como las variables empleadas, se describen a continuación. Nevo y Rosen (2012) demuestran que el método de emplear variables instrumentales que se suponen no están correlacionadas con las variables no observables, permite generar estimaciones útiles al utilizarse en funciones de demanda de mercados con productos diferenciados.

3 Ackerberg y Crawford (2009) muestran que el supuesto de exogeneidad en un modelo de demanda de bienes diferenciados no es necesario para obtener estimaciones de la elasticidad precio de la demanda consistentes, ya que esto va a depender de las propiedades de las variables instrumentales que se empleen en el análisis. 
Para validar los resultados del modelo, se aplicaron 2 pruebas sobre los resultados, la primera un contraste de Durbin (1954), Wu (1974) y Hausman (1978) para determinar si las variables deben ser tratadas como endógenas. Si el estadístico es significativo, se dice que las variables deben ser tratadas como endógenas. La segunda, una prueba de Sargan (1958) y Basmann (1960) para determinar si los instrumentos son válidos o no. Si el estadístico es significativo diremos que el (los) instrumentos no son válidos.

\subsection{Datos empleados}

A partir de la información suministrada por las firmas requeridas, y debido a la disponibilidad de la información, se decidió trabajar con las siguientes variables con periodicidad trimestral.

\section{ESTIMACIONES Y RESULTADOS}

Con el fin de estimar las elasticidades, así como el posible efecto en precios y en la concentración de mercado que pudiera derivarse de la integración empresarial entre la Firma 3 y la Firma 4 en el mercado de bebidas isotónicas, se propone el siguiente modelo econométrico:

$$
\text { (5) } \ln \left(\frac{s_{j}}{s_{0}}\right)=\beta_{0}-\alpha p_{j}+\xi_{j}
$$

Sin embargo, para modelar lo expuesto en la ecuación anterior (5), no se puede tomar directamente la variable de precios, en tanto como lo señala Berry (1994), podrían tenerse problemas de endogeneidad, que generarían estimadores sesgados e inconsistentes. Por lo anterior, se decide estimar el siguiente modelo econométrico con el uso de una variable instrumental:

$$
\text { (6) } \ln \left(\frac{s_{j}}{s_{0}}\right)=\beta_{0}-\alpha \tilde{p}_{j}+\xi_{j}
$$

Donde, $\tilde{p}_{j}$ corresponde a la variable de precios estimada a partir de la variable instrumental que se define como el precio unitario cancelado por cada firma para la adquisición de la botella PET de $500 \mathrm{ml}$ propuesta en la Tabla 2.

Adicionalmente, es importante señalar que en la medida en que la base contempla seis trimestres para cuatro firmas diferentes, se está trabajando en una estructura de datos panel, por lo que para la estimación econométrica se emplean las técnicas de 


\section{TABLA 2}

\section{VARIABLES EMPLEADAS}

\begin{tabular}{|c|c|c|c|}
\hline Variable & $\begin{array}{l}\text { Nombre } \\
\text { variable }\end{array}$ & Descripción & Fuente \\
\hline Mercado potencial & mkdopot & $\begin{array}{l}\text { Corresponde al promedio trimestral } \\
\text { del volumen en mililitros que } \\
\text { potencialmente se comprarían en } \\
\text { Colombia. Se calcula como el } \\
\text { porcentaje del total de la población } \\
\text { colombiana entre } 5 \text { y } 59 \text { años que } \\
\text { son consumidores potenciales de } \\
\text { bebidas isotónicas. Dicho porcentaje } \\
\text { se obtiene de un estudio de Nielsen. }\end{array}$ & $\begin{array}{l}\text { Estimación del GIE a partir } \\
\text { de Nielsen. }\end{array}$ \\
\hline $\begin{array}{l}\text { Precio por mililitro } \\
\text { de la bebida } \\
\text { isotónica }\end{array}$ & $p r_{-} r$ & $\begin{array}{l}\text { Corresponde al promedio trimestral } \\
\text { del precio por mililitro de la bebida } \\
\text { isotónica de cada marca objeto de } \\
\text { análisis. Con el fin de tener una } \\
\text { presentación de referencia y en } \\
\text { función de la información reportada } \\
\text { en las visitas, se tomó la presentación } \\
\text { de } 500 \mathrm{ml} \text { PET, para las diferentes } \\
\text { firmas. Este precio se expresa en } \\
\text { términos reales a diciembre de } 2013 \text {. }\end{array}$ & $\begin{array}{l}\text { Requerimiento solicitado a } \\
\text { las firmas GIE-SIC }\end{array}$ \\
\hline Ventas en volumen & $q u$ & $\begin{array}{l}\text { Cantidad vendida en mililitros de } \\
\text { bebidas isotónicas por firma. }\end{array}$ & $\begin{array}{l}\text { Requerimiento solicitado a } \\
\text { las firmas GIE-SIC }\end{array}$ \\
\hline "Outside good" & $X$ & $\begin{array}{l}\text { Se construyó como la diferencia entre } \\
\text { el mercado potencial y las ventas } \\
\text { totales en mililitros (trimestral), } \\
\text { con el fin de representar el número } \\
\text { de personas que toman la decisión } \\
\text { de no comprar esas bebidas en dicho } \\
\text { trimestre. Lo anterior se expresa } \\
\text { en términos de participación del } \\
\text { mercado, para lo cual se divide por } \\
\text { el mercado potencial. }\end{array}$ & $\begin{array}{l}\text { Elaboración GIE y } \\
\text { GEE (SIC) a partir de la } \\
\text { información entregada por } \\
\text { las firmas }\end{array}$ \\
\hline $\begin{array}{l}\text { Participación de } \\
\text { mercado }\end{array}$ & $X$ & $\begin{array}{l}\text { Corresponde al cociente entre } \\
\text { las ventas anuales del vehículo } \\
\text { (producto) } j \text { y el mercado potencial } \\
\text { de ese año. }\end{array}$ & $\begin{array}{l}\text { Cálculos propios a partir de } \\
\text { la información entregada por } \\
\text { las firmas }\end{array}$ \\
\hline Variables Dummy & $d i$ & Dummies de tiempo & \\
\hline Instrumento & pr_botella_r $r$ & $\begin{array}{l}\text { Corresponde al precio unitario } \\
\text { cancelado por cada firma para la } \\
\text { adquisición del insumo (botella } \\
500 \mathrm{ml} \text { PET). }\end{array}$ & $\begin{array}{l}\text { Elaboración GIE y } \\
\text { GEE (SIC) a partir de la } \\
\text { información entregada por } \\
\text { las firmas }\end{array}$ \\
\hline
\end{tabular}

* GEE: Grupo de Estudios Económicos y GIE: Grupo de Integraciones Empresariales.

Superintendencia de Industria y Comercio.

Fuente: Elaboración GEE-SIC. 
efectos fijos y efectos aleatorios con el fin de validar la existencia o no del componente idiosincrático no observado en el término de error.

Este instrumento puede considerarse adecuado pues, en primer lugar, la botella PET es el principal insumo y por ende costo que enfrentan los productores de bebidas isotónicas por lo que de su precio dependerá el precio final de la bebida. Adicionalmente, es posible establecer que este precio es independiente de las cantidades de bebidas isotónicas vendidas en el mercado en cuanto a que la compra de este insumo por parte de las productoras de bebidas isotónicas representa tan solo una cantidad marginal comparada con las cantidades de botellas PET 500ml requeridas como insumo principal en la producción de otras bebidas. En particular, usando información de la base de datos Passport de Euromonitor International, es posible establecer que el número de botellas de bebidas deportivas y energizantes, categoría que incluye bebidas energizantes no relevantes en el presente ejercicio, representó en 2013 aproximadamente el 3,4\% del total de botellas de otras bebidas ${ }^{4}$ en presentaciones PET de $500 \mathrm{ml}$ por lo cual las cantidades demandadas únicamente en el mercado de bebidas isotónicas tendrían un efecto nulo sobre el precio de las botellas. Por tal razón, y como será confirmado más adelante, el precio de las botellas PET $500 \mathrm{ml}$ puede ser utilizado como instrumento en el modelo estimado a continuación.

El primer propuesto corresponde a la estimación de la ecuación (6) empleado la variable el precio por unidad cancelado por cada firma por concepto de la adquisición del insumo denominado botellas PET 500ml como instrumento. Los resultados de esta estimación se obtuvieron siguiendo la metodología propuesta por Björnerstedt y Verboven (2013) y se presentan en la Tabla 3.

$$
\text { (7) } \ln \left(\frac{s_{j}}{s_{0}}\right)=\beta_{0}-\alpha \tilde{p}_{j}+\xi_{j}
$$

Como se mencionó en la sección de metodología, se aplicaron dos pruebas para determinar si las variables son exógenas y si el instrumento es válido. A continuación, se presentan los resultados de las pruebas.

\begin{tabular}{|l|c|c|}
\hline \multicolumn{3}{|c|}{ Pruebas de restricción de identificación (valides de los instrumentos) } \\
\hline & Estadístico & Valor Probabilístico \\
\hline Sargan (score) chi2(3) & 4.2877 & $(\mathrm{p}=0.2357)$ \\
Basmann chi2(3) & 4.8294 & $(\mathrm{p}=0.1956)$ \\
\hline
\end{tabular}

4 Otras bebidas comprenden las siguientes categorías: bebidas carbonatadas, agua embotellada y bebidas de té listas para consumo. 


\section{TABLA 3}

RESULTADOS ECONOMETRICOS DEL MODELO

\begin{tabular}{|c|c|}
\cline { 2 - 2 } \multicolumn{1}{c|}{} & $\begin{array}{c}\text { Variables Instrumentales } \\
\text { (Efectos Fijos - Within) } \\
\text { Var Dep: } M \_l s \\
\text { Errores estándar entre }()\end{array}$ \\
\hline Cons & -3.304241 \\
& $(0.1767)^{* *} r$ \\
& -0.6363524 \\
Wald Chi2(1) & $(0.0840)^{* * *}$ \\
Prob & 44629.09 \\
sigma_u & 0.000 \\
sigma_e & 1.5223 \\
rho & 0.1075 \\
Prueba $F\left(u \_i=0\right)$ & 0.9950 \\
F(3,19) & \\
Prob $>$ F & 198.81 \\
Variable instrumentada & 0.000 \\
Instrumento & pr_r $r$ \\
Prueba de relevancia de la & pr_botellas_r $r$ \\
primera etapa & \\
Estadístico $F$ & \\
Prob $(F)$ & 19.94 \\
\hline
\end{tabular}

Fuente: cálculos GEE-SIC.

\begin{tabular}{|c|c|c|}
\hline \multicolumn{3}{|c|}{$\begin{array}{l}\text { Prueba de Endogeneidad } \\
\text { Ho: variables son exógenas }\end{array}$} \\
\hline & Estadístico & Valor Probabilístico \\
\hline $\begin{array}{l}\text { Durbin (score) chi2(1) } \\
\text { Wu-Hausman F(1,46) }\end{array}$ & $\begin{array}{l}2.8473 \\
3.9067\end{array}$ & $\begin{array}{l}(\mathrm{p}=0.5803) \\
(\mathrm{p}=0.4602)\end{array}$ \\
\hline
\end{tabular}

Ambas pruebas determinan que, primero las variables del modelo pueden ser tratadas como exógenas, y segundo que el instrumento utilizado es válido en la estimación. La hipótesis nula de ambas pruebas no puede ser rechazada al 5\% de significancia. Adicionalmente, en la Tabla 3 se presenta un contraste de relevancia del instrumento a través de una prueba F sobre los instrumentos en la primera etapa de la estimación, el cual permite concluir que los coeficientes son conjuntamente significativos en la primera etapa, evidenciando que el instrumento es relevante. 
A partir de la estimación presentada en la Tabla 3, que revela que los parámetros estimados presentan el signo adecuado y que son independiente y conjuntamente significativos a un nivel de significancia del 5\% $(\alpha=0.05)$, se procede a calcular el valor estimado de las elasticidades propias y cruzadas (Tabla 4) aplicando la metodología de Berry (1994) y cuyas ecuaciones se presentaron en las ecuaciones (2) y (3) de la subsección Metodología y Datos.

TABLA 4

ESTIMACION DE ELASTICIDADES

\begin{tabular}{|c|c|c|c|c|}
\hline Valor Alpha & -0.636 & & & \\
\hline & Media & $\begin{array}{c}\text { Desviación } \\
\text { Estándar }\end{array}$ & Mínimo & Máximo \\
\hline $\begin{array}{c}\text { Elásticidad precio } \\
\text { M_ejj } \\
\begin{array}{c}\text { Elásticidad precio cruzada } \\
\text { M_ejk }\end{array}\end{array}$ & -1.254 & 0.463 & -1.975 & -0.815 \\
\hline
\end{tabular}

Fuente: Cálculos GEE-SIC.

De esa forma, según los resultados de la Tabla 4 se encuentra que, ante variaciones en el precio propio, la demanda de las bebidas isotónicas analizadas en la muestra responde más que proporcionalmente al encontrarse una elasticidad propia mayor a 1 en términos absolutos, es decir que es muy sensible a cambios en el precio. Por su parte, la elasticidad cruzada, que daría cuenta de la respuesta de la demanda del bien $j$ ante variaciones del precio del bien $k$, se encuentra una variación reducida al hallar una elasticidad cruzada de 0.026 , lo cual indica una reacción inelástica, es decir que es poco insensible a cambios en el precio del bien $k$. De esa forma, la elasticidad cruzada estimada en el modelo estaría señalando una reducida presión competitiva que podría ejercer un productor al reducir los precios sobre la demanda de otro productor. En particular, por cada $1 \%$ que se incremente el precio de las bebidas isotónicas distintas a Marca 3, se estima un incremento de $0.026 \%$ en la cantidad vendida de este último producto.

Con el fin de valorar la razonabilidad económica de las elasticidades de precio propia y precio cruzada encontradas en el ejercicio, la Tabla 5 que se presenta a continuación resume los precios promedio y participaciones de mercado de cada una de las marcas consideradas en el periodo de tiempo utilizado en la estimación.

De la Tabla 5 anterior es posible concluir que, dado que la Marca 3 presenta el precio promedio más alto, 3.197, en conjunto con la mayor participación de mercado, $83.3 \%$, es razonable encontrar que la elasticidad precio cruzada con las otras marcas 


\section{TABLA 5}

PRECIOS PROMEDIO Y PARTICIPACIONES DE MERCADO

\begin{tabular}{|c|c|c|}
\hline id_marca & $\begin{array}{c}\text { Precio } \\
\text { promedio }\end{array}$ & $\begin{array}{c}\text { Participación de } \\
\text { mercado }\end{array}$ \\
\hline 1 & 1.488 & $4.34 \%$ \\
2 & 1.575 & $5.65 \%$ \\
3 & 3.197 & $83.3 \%$ \\
4 & 1.786 & $6.71 \%$ \\
\hline
\end{tabular}

Fuente: cálculos GEE-SIC.

sea muy cercana a cero, es decir que ante cambios en el precio de la marca 3 la demanda por las demás marcas no cambie significativamente, tal y como se estimó en el modelo presentado anteriormente.

Otro ejercicio que permite tener un contrafactual para establecer la razonabilidad de los resultados encontrados consistió en calcular de manera preliminar las elasticidades utilizando estimaciones lineales y logarítmicas a partir de los datos inicialmente consolidados para el ejercicio de precios y cantidades vendidas. Los resultados obtenidos fueron los siguientes presentados en la siguiente Tabla $6^{5}$.

\section{TABLA 6}

ELASTICIDAD PRECIO PROPIO Y CRUZADA PROMEDIO A PRIORI

\begin{tabular}{|l|c|c|}
\hline \multicolumn{1}{|c|}{ Modelo } & $\begin{array}{c}\text { Elasticidad Propia } \\
\text { Promedio }\end{array}$ & $\begin{array}{c}\text { Elasticidad Cruzada } \\
\text { respecto de la marca 3 }\end{array}$ \\
\hline Lineal & -1.5707 & 0.0375 \\
Logarítmico & -1.4842 & 0.0352 \\
\hline
\end{tabular}

Fuente: cálculos GEE-SIC.

De acuerdo con los resultados presentados en la Tabla 6 , se confirma la validez de los resultados obtenidos mediante la estimación del modelo logit anidado con la metodología descrita.

5 El ejercicio presentado en esta tabla constituye una aproximación básica a las elasticidades, y solo se debe entender como medio de contraste para validar la razonabilidad económica de los resultados encontrados. En este sentido, el lector no debe esperar prueba alguna de robustez de los modelos que permitieron llegar a las estimaciones presentadas en la misma. 
Ante estos niveles de elasticidad precio propia y cruzada de la demanda, el resultado esperado de la simulación de la integración entre la Firma 3 y la Firma 4 sería el siguiente presentado a continuación en la Tabla 7.

TABLA 7

EFECTO ESPERADO SOBRE PRECIOS A PARTIR DEL MODELO

\begin{tabular}{|c|c|c|c|}
\hline id_marca & Pre-Integración & Post-Integración & Cambio Relativo \\
\hline 1 & 1.488 & 1.488 & 0.000 \\
2 & 1.575 & 1.575 & 0.000 \\
3 & 3.197 & 3.207 & 0.003 \\
4 & 1.786 & 1.855 & 0.040 \\
\hline
\end{tabular}

Fuente: cálculos GEE-SIC.

De esa forma, se encuentra que las Firmas 1 y 2, no tendrían incentivos a incrementar los precios, mientras que la Firma 3 y Firma 4 podrían reportar incrementos en precios, pero que se ubicarían por debajo del $4 \%$.

Lo anterior, permitiría concluir que la integración no generaría una presión importante al alza en precios, como resultado de una elasticidad precio cruzada reducida y una alta elasticidad precio propio de la demanda.

\section{CONSIDERACIONES FINALES Y CONCLUSIONES}

Partiendo de la importancia que la herramienta de simulación de integraciones empresariales ha obtenido, y el rápido crecimiento que su implementación ha adquirido a nivel mundial. Este documento sintetizó el análisis del mercado de bebidas isotónicas en torno a la integración empresarial propuesta en el año 2014 entre dos grandes empresas productoras de bebidas istónicas, mediante la estimación de un modelo microeconométrico simulando la integración, y siguiendo la metodología propuesta por Berry (1994) y Björnerstedt y Verboven (2013). En particular, se evaluó el posible impacto sobre los precios y la competencia que pudiera derivarse de la integración en el mercado de bebidas isotónicas en Colombia, particularmente entre los productos de consumo de cada una de las intervinientes.

Tras el análisis descriptivo y el ejercicio econométrico planteado, es posible concluir que no se encuentran indicios sobre un posible efecto explotativo explicado por la inexistencia de presiones al alza en precios como resultado de la posible integración.

Lo anterior, se explica en cuanto el producto Marca 3 parece presentar un comportamiento independiente a lo reportado por los otros productos analizados 
puesto que mientras estos últimos mostraron importantes variaciones, el precio por mililitro de la Marca 3 se mantuvo estable.

Asimismo, se encontró que la elasticidad precio propio de la demanda es mayor a 1, lo que indica que la demanda de los productos analizados es sensible ante las variaciones de los precios. Sin embargo, la demanda del bien no responde de igual forma ante la variación de los precios de otro bien, puesto que la elasticidad cruzada entre el bien $j$ y el bien $k$ fue cercana a 0 .

Estos resultados entonces sugerirían que la integración no tendría un efecto significativo en el caso del mercado de bebidas isotónicas en Colombia, básicamente porque la integración no generaría una presión importante al alza en precios, como resultado de una elasticidad precio cruzada reducida y una alta elasticidad precio propio de la demanda.

El documento deja un precedente en Colombia a efectos de utilizar herramientas microeconométricas en el análisis de integraciones empresariales que realiza la Superintendencia de Industria y Comercio, autoridad de competencia colombiana. Nuevas aplicaciones de esta metodología contribuirán a futuro definir de manera objetiva el análisis de sustituibilidad en materia de definición de mercados relevantes en diferentes sectores.

\section{REFERENCIAS}

ACKERBERG, D. y CRAWFORD, G. (2009). "Estimating Price Elasticities in Differentiated Product Demand Models with Endogenous Characteristics". Working Paper, pp. 22.

BASMANN, R. (1960). "On finite sample distributions of generalized classical linear identifiability test statistics". Journal of the American Statistical Association, Vol. 55: 650-659.

BERRY, S. (1994). "Estimating Discrete-Choice Models of Product Differentiation". The RAND Journal of Economics, Vol. 25(2), 242-262.

BJÖRNERSTEDT, J. and VERBOVEN. F. (2016). "Does Merger Simulation Work? Evidence from the Swedish Analgesics Market". American Economic Journal: Applied Economics, 8(3):125-64.

BJÖRNERSTEDT, J. y VERBOVEN, F. (2016). "Merger Simulation with Nested Logit”, The Stata Journal, Vol. 14, Numero 3, pp. 511-540.

BUDZINSKI, O. y RUHMER, I. (2009). "Merger Simulation in Competition Policy: A Survey", Journal of Competition Law and Economics, Vol. 6 (2), pp. 277-319.

DEATON, A. y MUELLBAUER J. (1980). "An Almost Ideal Demand System", The American Economic Review, Vol. 70, No. 3.

DE BETTIGNIES, J. y ROSS, T. (2014). "Mergers, Agency Costs, and Social Welfare", Journal of Law, Economics, and Organization, Vol. 30 (2), pp. 401-436.

DURBIN, J. (1954). "Errors in variables". Review of the International Statistical Institute, Vol. 22: pp. 23-32.

EPSTEIN R. and RUBINFELD, D. (2001). "Merger Simulation: A Simplified Approach with New Applications", Vol. 69, Antitrust L.J. 883. Available at: http://scholarship.law.berkeley.edu/facpubs/1362

FARRELL, J. y SHAPIRO, C. (1990). "Horizontal Mergers: An Equilibrium Analysis", The American Economic Review, Vol. 80 (1), pp. 107-126.

FROEB, L., TSCHANTZ, S. y WERDEN, G. (2003). "Bertrand Competition with Capacity Constraints: Mergers Among Parking Lots", Journal of Econometrics, Vol. 113, pp. 49-67.

FROEB, L. y WERDEN, G. (1994). "The Effects of Mergers in Differentiated Products Industries: Logit Demand and Merger Policy", Journal of Law, Economics, and Organization, Vol. 10 (2), pp. 407-426. 
HAUSMAN, J. (1978). "Specification tests in econometrics". Econometrica, Vol. 46: 1251-1271.

XIVALDI, M. y VERBOVEN F. (2005). "Quantifying the effects from horizontal mergers in European competition policy", International Journal of Industrial Organization, Vol. 23 (9), pp. 669-691.

NEVO, A. (2000). "Mergers with Differentiated Products: the Case of the Ready-to-Eat Cereal Industry", RAND Journal of Economics, 31(3), 395-421.

NEVO, A. and ROSEN, A. (2012). "Identification with Imperfect Instruments", The Review of Economics and Statistics, Vol. 94, No. 3, Pages: 659-671.

SANCHEZ, D. (2013). "Análisis De Elasticidades En El Mercado Automotor Colombiano (2009-2011) Mediante Un Modelo Logit Anidado". Disponible en: http://ssrn.com/abstract=2248003 o http:// dx.doi.org/10.2139/ssrn.224800312

SARGAN, J. (1958). "The estimation of economic relationships using instrumental variables". Econometrica, Vol. 26: 393-415.

WU, D. (1974). "Alternative tests of independence between stochastic regressors and disturbances: Finite sample results". Econometrica, Vol. 42: pp. 529-546. 